\title{
A first case of ductal adenocarcinoma of the prostate having characteristics of neuroendocrine phenotype with PTEN, RB1 and TP53 alterations
}

Hiroaki Kobayashi ${ }^{1}$, Takeo Kosaka ${ }^{1 *} \mathbb{0}$, Kohei Nakamura² ${ }^{2}$ Kazunori Shojo ${ }^{1}$, Hiroshi Hongo ${ }^{1}$, Shuji Mikami ${ }^{3}$, Hiroshi Nishihara ${ }^{2}$ and Mototsugu Oya ${ }^{1}$

\begin{abstract}
Background: Ductal adenocarcinoma and neuroendocrine cancer are rare subtypes of prostate cancer with poor prognosis and limited therapeutic options. We present the first case of ductal adenocarcinoma having a neuroendocrine phenotype.

Case presentation: A 63-year-old man presented with gross hematuria and urinary retention, and his serum prostate-specific antigen level was $4.58 \mathrm{ng} / \mathrm{mL}$. We performed transurethral resection of the prostate, and the diagnosis was ductal adenocarcinoma with a Gleason score of $5+4$ for acinar adenocarcinoma. Magnetic resonance imaging showed local invasion of left lobe of the prostate and bone metastasis of the left trochanteric section of the femur. Multidisciplinary treatments such as androgen deprivation therapy, chemoradiation therapy, and surgery for metastatic lesions have led to long-term survival. Since next-generation sequencing revealed PTEN and RB1 co-loss and TP53 mutations, we re-evaluated the immunohistochemistry and he was found to be positive for synaptophysin.
\end{abstract}

Conclusions: This is the first Japanese case of ductal adenocarcinoma with a neuroendocrine phenotype. Genetic analysis may help not only guide the therapeutic strategies, but also sometimes with the diagnosis.

Keywords: Ductal adenocarcinoma, Neuroendocrine prostate cancer, Next-generation sequencing, Case report

\section{Background}

Ductal adenocarcinoma (DCa) of the prostate is a rare morphologic subtype, occurring in less than $1 \%$ and up to $5 \%$ in cases of acinar adenocarcinoma (ACa), defined histologically by elongated cells and papillary or cribriform architecture [1-3]. Since DCa often presents at an advanced clinical stage with local or distant metastases and no effective treatment has been established, the prostate-specific mortality is significantly worse than

\footnotetext{
*Correspondence: takemduro@gmail.com

${ }^{1}$ Department of Urology, Keio University School of Medicine, 35

Shinanomachi, Shinjuku-ku, Tokyo 160-8582, Japan

Full list of author information is available at the end of the article
}

that of $\mathrm{ACa}$ [1]. In contrast, neuroendocrine prostate cancer (NEPC) is an extremely rare entity with poor prognosis and limited therapeutic options [4]. The pathological characteristics of NEPC include staining with immunohistochemical markers (CD56, synaptophysin, chromogranin A, and NSE), high proliferative rate (Ki67 >50\%), and the presence of TMPRSS2-ERG rearrangement. Synaptophysin is the most sensitive marker, NSE is also sensitive but not specific, and chromogranin A is the most specific marker [5, 6]. Little is known regarding the genetic and specific histopathological characteristics of DCa and NEPC, and to date, few studies have investigated the genetic profile of these tumors. 
Therefore, the accumulation of case reports and genetic or histopathological analyses is expected to add to our understanding to this disease entity. Herein, we report a case of a DCa mixed with ACa with a neuroendocrine phenotype.

\section{Case presentation}

A 63-year-old man presented with gross hematuria and urinary retention. He had no remarkable medical or family history. His serum prostate-specific antigen (PSA) was $4.58 \mathrm{ng} / \mathrm{mL}$, and urine cytology was class 3 (atypical urothelial cells). Since cystoscopy revealed a papillary tumor in the prostatic urethra, we performed transurethral resection of the prostate. The pathological diagnosis was poorly differentiated adenocarcinoma with Gleason score of $5+4=9$ with DCa (Fig. 1). Magnetic resonance imaging showed local invasion of the left lobe of the prostate and bone metastasis of the left trochanteric section of the femur (Fig. 2). Although he was treated with androgen deprivation therapy (ADT), 10 months later, the disease became castration-resistant prostate cancer. At this point, the PSA level was $3.66 \mathrm{ng} / \mathrm{mL}$ and serum NSE was $9.6 \mathrm{ng} / \mathrm{mL}$. Then, we administered three courses of docetaxel $\left(75 \mathrm{mg} / \mathrm{m}^{2}\right)$ as the second-line therapy. Since the serum PSA level was decreased to $0.54 \mathrm{ng} / \mathrm{mL}$, the patient was treated with a combination of $160 \mathrm{mg}$ of enzalutamide and intensity-modulated radiation therapy (IMRT) consisting of 78 Gy administered in 39 fractions for the prostate and 40 Gy administered in 14 fractions for the left femur as the third-line therapy, which decreased the PSA level to be nadir of $0.03 \mathrm{ng} / \mathrm{mL}$. After the combination therapy, enzalutamide was continued for 13 months, and when the serum PSA increased to $0.35 \mathrm{ng} / \mathrm{mL}$, four courses of cabazitaxel $\left(25 \mathrm{mg} / \mathrm{m}^{2}\right)$ were administered as the fourth-line therapy. Despite multimodal therapy, positron emission tomography/ computed tomography (PET/CT) showed viability of the left trochanteric section of the femur. Thus, we performed left femoral head replacement, and we confirmed the absence of viable cancer cells pathologically. After 1 year, he was treated with local radiation of 60 Gy administered in 25 fractions because the PSA level increased to $0.76 \mathrm{ng} / \mathrm{mL}$ and pelvic lymph node metastasis was noted. At this point, we performed targeted genome sequencing of the tumor specimen from the transurethral resection and re-evaluated the immunochemistry findings. We identified PTEN p. R233fs*10, RB1 loss exons 18-27, and TP53 p.R249G mutations by genome sequencing (Table 1), and the patient was found to be positive for synaptophysin by immunohistochemistry (Fig. 1F). Currently, he is undergoing chemotherapy with cabazitaxel, and his PSA/NSE level was around 0.20/9.0 ng/mL.

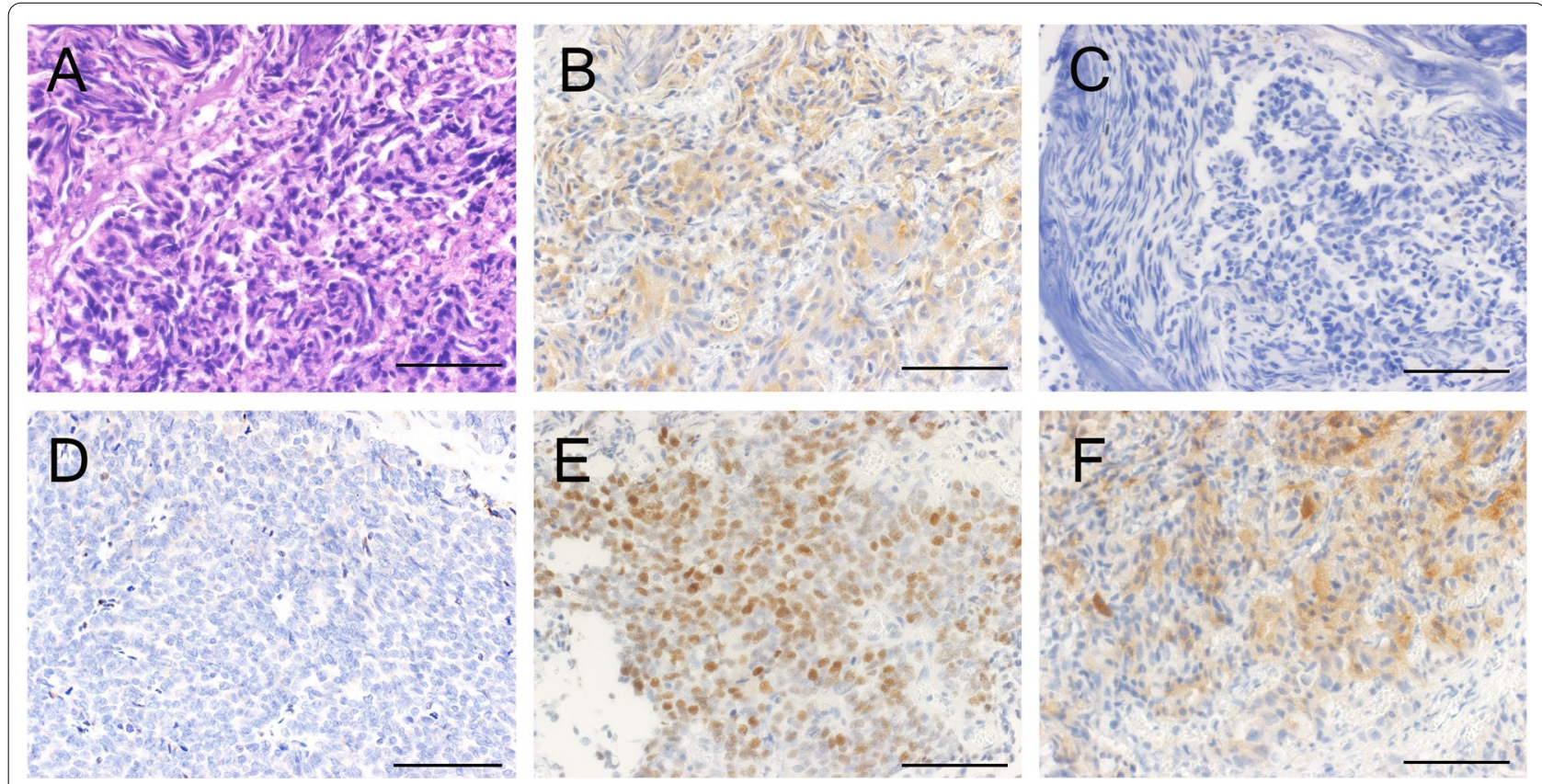

Fig. 1 Representative images of $\mathbf{A}$ hematoxylin and eosin staining and B PSA, C loss of PTEN, D loss of RB1, E TP53 and F synaptophysin immunohistochemical staining of transurethral resection of prostate specimens. $B a r=0.1 \mathrm{~mm}$ 


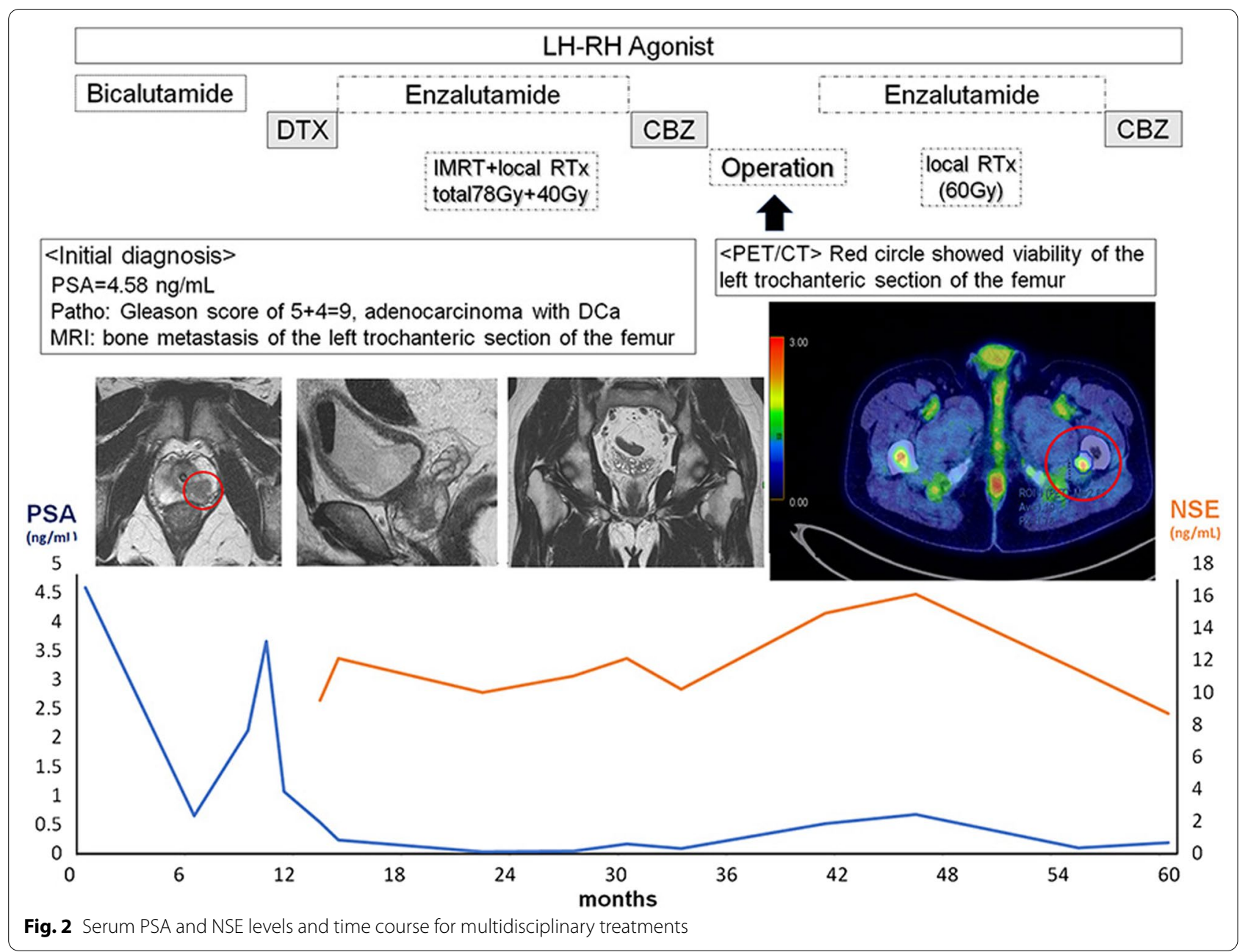

Table 1 Results of FoundationOne ${ }^{\circledR}$ companion diagnostic (F1CDx) assay

\begin{tabular}{llll}
\hline Biomarker findings & \multicolumn{2}{l}{ Genomic findings } \\
\hline MSI & Cannot be determined & PTEN & $\begin{array}{l}\text { R233fs*10, splice site } \\
\text { 480_492+2delCAGAGACAA }\end{array}$ \\
& & AAAGGT \\
TMB $\quad 3$ Muts/Mb & RB1 & LOSS \\
& & TP53 & R249G
\end{tabular}

MSI, microsatellite instability; TMB, tumor mutational burden

\section{Discussion and conclusions}

Patients with metastatic ACa are currently best treated with palliative drug treatments, such as ADT plus androgen receptor-targeted agents or chemotherapy [7]. Recent studies have demonstrated that early detection and aggressive treatment of metastatic lesions with surgery or radiation therapy appears to be a feasible strategy in patients with newly diagnosed oligometastatic $\mathrm{ACa}$
[8-10]. On the other hand, DCa has no established treatment strategy, and palliative ADT or chemotherapy alone cannot be expected to have a long-term prognosis even though a significantly greater frequency of men with DCa had local or distant metastasis at diagnosis. In our case, the patient was aggressively treated with ADT and chemotherapy with IMRT and local radiation therapy, while the initial diagnosis was a DCa with a Gleason score of $5+4=9$ of $\mathrm{ACa}$ and bone metastasis. When PET/CT revealed revitalization of the left trochanteric section of the femur, we decided to perform the operation and confirmed the absence of viable cancer cells. Based on these aggressive multidisciplinary treatments made for the long-term survival.

Currently, in Japan, we can perform genome sequencing of tumor specimens in order to find the next treatment target drug in rare cancer patients in which the typical treatment effect becomes less remarkable. In this case, we identified PTEN and RB1 co-loss and TP53 mutations by next-generation sequencing. PTEN loss, RB1 loss, 
and TP53 mutations are common genomic alterations in many cancers. Vinceneux et al. reported that a complete loss of PTEN expression was more frequent in DCa (34\%) than in $\mathrm{ACa}(11 \%)$ by immunohistochemistry [11]. Some reports have also indicated that PTEN loss occurring in DCa is associated with multiple markers of poor prognosis and has recently been associated with cribriform morphology, similar to that of ductal histology [12-14]. In fact, there were some scattered areas of complete loss of PTEN expression (Fig. 1C). Similarly, loss of function of the tumor suppressive function of TP53 and RB1 are the common mutant genes in $\mathrm{ACa}$ and are associated with aggressive tumor progression or metastatic ACa [15-17], while there was no confident evidence about the correlation between RB1 or TP53 mutation and DCa. Tarjan et al. reported that the combination of TP53 and chromogranin A expression in cancer cells is associated with ductal differentiation of $\mathrm{ACa}$ [18]. On the other hand, we performed immunohistochemistry for chromogranin A and synaptophysin staining, and the patient was found to be positive for synaptophysin (Fig. 1F) because these three genomic mutations are often found in patients with NEPC [19]. These results indicated that we could have the treatment option to perform the administration of a platinum agent for the future.

In conclusion, to the best of our knowledge, there are few case reports of genomic analysis for DCa, which is the first Japanese report of DCa with PTEN and RB1 coloss and TP53 mutation. Genetic analysis may help not only guide the therapeutic strategies, but also sometimes with the diagnosis.

\section{Abbreviations}

DCa: Ductal adenocarcinoma; ACa: Acinar adenocarcinoma; NEPC: Neuroendocrine prostate cancer; PSA: Prostate-specific antigen; ADT: Androgen deprivation therapy; IMRT: Intensity modulated radiation therapy; PET/CT: Positron emission tomography/ computed tomography.

\section{Acknowledgements}

Yoko Suzuki provided technical assistance.

\section{Authors' contributions}

Conception and design: TK, HN, and MO. Acquisition and analysis of data: TK $\mathrm{KN}, \mathrm{KS}, \mathrm{HH}$, and HN. Drafting the manuscript and figures: HK, TK, SM, HN, and MO. All authors read and approved the final manuscript.

\section{Funding}

None.

\section{Availability of data and materials}

The datasets used and analyzed in this study are available from the corresponding author on reasonable request.

\section{Declarations}

Ethics approval and consent to participate

This study was approved by the Ethical Committee of Keio University Hospital (\#20160084)

\section{Consent for publication}

Written informed consent was obtained from the patient for publication of this case report. A copy of the consent form is available for review by the Editor of this journal.

\section{Competing interests}

The authors declare that they have no competing interests.

\section{Author details}

${ }^{1}$ Department of Urology, Keio University School of Medicine, 35 Shinanomachi, Shinjuku-ku, Tokyo 160-8582, Japan. ${ }^{2}$ Genomics Unit, Keio Cancer Center, Keio University School of Medicine, Tokyo, Japan. ${ }^{3}$ Division of Diagnostic Pathology, Keio University School of Medicine, Tokyo, Japan.

Received: 27 May 2021 Accepted: 29 September 2021

Published online: 09 October 2021

\section{References}

1. Morgan TM, Welty CJ, Vakar-Lopez F, Lin DW, Wright JL. Ductal adenocarcinoma of the prostate: increased mortality risk and decreased serum prostate specific antigen. J Urol. 2010;184(6):2303-7.

2. Meeks JJ, Zhao LC, Cashy J, Kundu S. Incidence and outcomes of ductal carcinoma of the prostate in the USA: analysis of data from the surveillance, epidemiology, and end results program. BJU Int. 2012;109(6):831-4.

3. Seipel AH, Delahunt B, Samaratunga H, Egevad L. Ductal adenocarcinoma of the prostate: histogenesis, biology and clinicopathological features. Pathology. 2016;48(5):398-405.

4. Epstein Jl, Amin MB, Beltran H, Lotan TL, Mosquera JM, Reuter VE, et al. Proposed morphologic classification of prostate cancer with neuroendocrine differentiation. Am J Surg Pathol. 2014;38(6):756-67.

5. Kranitz N, Szepesvary Z, Kocsis K, Kullmann T. Neuroendocrine cancer of the prostate. Pathol Oncol Res. 2020;26(3):1447-50.

6. Parimi V, Goyal R, Poropatich K, Yang XJ. Neuroendocrine differentiation of prostate cancer: a review. Am J Clin Exp Urol. 2014;2(4):273-85.

7. Cornford $P$, van den Bergh RCN, Briers E, van den BroeckT, Cumberbatch MG, De Santis M, et al. EAU-EANM-ESTRO-ESUR-SIOG guidelines on prostate cancer part II-2020 update: treatment of relapsing and metastatic prostate cancer. Eur Urol. 2021;79(2):263-82.

8. Heidenreich A, Pfister D, Porres D. Cytoreductive radical prostatectomy in patients with prostate cancer and low volume skeletal metastases: results of a feasibility and case-control study. J Urol. 2015;193(3):832-8.

9. O'Shaughnessy MJ, McBride SM, Vargas HA, Touijer KA, Morris MJ, Danila DC, et al. A pilot study of a multimodal treatment paradigm to accelerate drug evaluations in early-stage metastatic prostate cancer. Urology. 2017;102:164-72.

10. Parikh RR, Byun J, Goyal S, Kim IY. Local therapy improves overall survival in patients with newly diagnosed metastatic prostate cancer. Prostate. 2017:77(6):559-72.

11. Vinceneux A, Bruyere F, Haillot O, Charles T, de la Taille A, Salomon L, et al. Ductal adenocarcinoma of the prostate: clinical and biological profiles. Prostate. 2017;77(12):1242-50

12. Gillard M, Lack J, Pontier A, Gandla D, Hatcher D, Sowalsky AG, et al. Integrative genomic analysis of coincident cancer foci implicates CTNNB1 and PTEN alterations in ductal prostate cancer. Eur Urol Focus. 2019:5(3):433-42.

13. Jeong SU, Kekatpure AK, Park JM, Han M, Hwang HS, Jeong HJ, et al. Diverse immunoprofile of ductal adenocarcinoma of the prostate with an emphasis on the prognostic factors. J Pathol Transl Med. 2017:51(5):471-81.

14. Ronen S, Abbott DW, Kravtsov O, Abdelkader A, Xu Y, Banerjee A, et al. PTEN loss and p27 loss differ among morphologic patterns of prostate cancer, including cribriform. Hum Pathol. 2017;65:85-91.

15. Arora K, Barbieri CE. Molecular subtypes of prostate cancer. Curr Oncol Rep. 2018;20(8):58.

16. Thangavel C, Boopathi E, Liu Y, Haber A, Ertel A, Bhardwaj A, et al. RB loss promotes prostate cancer metastasis. Can Res. 2017;77(4):982-95.

17. Ku SY, Rosario S, Wang Y, Mu P, Seshadri M, Goodrich ZW, et al. Rb1 and Trp53 cooperate to suppress prostate cancer lineage plasticity, metastasis, and antiandrogen resistance. Science. 2017;355(6320):78-83. 
18. Tarjan M, Lenngren A, Hellberg D, Tot T. Immunohistochemical verification of ductal differentiation in prostate cancer. APMIS. 2012;120(6):510-8.

19. Kaur H, Samarska I, Lu J, Faisal F, Maughan BL, Murali S, et al. Neuroendocrine differentiation in usual-type prostatic adenocarcinoma: Molecular characterization and clinical significance. Prostate. 2020;80(12):1012-23.

\section{Publisher's Note}

Springer Nature remains neutral with regard to jurisdictional claims in published maps and institutional affiliations.
Ready to submit your research? Choose BMC and benefit from:

- fast, convenient online submission

- thorough peer review by experienced researchers in your field

- rapid publication on acceptance

- support for research data, including large and complex data types

- gold Open Access which fosters wider collaboration and increased citations

- maximum visibility for your research: over 100M website views per year

At BMC, research is always in progress.

Learn more biomedcentral.com/submissions 